\title{
A SINGLE SESSION OF ACTIVE VIDEO GAME PLAY PROMOTES POST-EXERCISE HYPOTENSION IN HYPERTENSIVE MIDDLE-AGED SUBJECTS
}

original paper

( ) University School of Physical Education in Wroclaw

DOI: https://doi.org/10.5114/hm.2018.74063

\section{TAIS FEITOSA DA SILVA, ANA CARLA LIMA DE FRANCA, MARIZANGELA FERREIRA DE SOUZA, ALEXANDRE SERGIO SILVA}

Federal University of Paraíba, Paraíba, Brazil

\begin{abstract}
Purpose. To determine whether an active video game session promoted post-exercise hypotension, similar to walking.

Methods. Fifteen hypertensive subjects ( $49.0 \pm 1.6$ years) of both genders performed five randomized sessions, lasting 60 minutes: 1) active video game; 2) sedentary video game; 3) walking on a treadmill with moderate intensity; 4) walking on a treadmill with similar intensity to the active video game; and 5) control without exercise. Blood pressure and cardiac autonomic modulation measurements were taken at rest and every 10 minutes post exercise for 60 minutes.

Results. The active video game promoted a reduction in systolic blood pressure $(-14.4 \pm 3.0 \mathrm{mmHg}, p<0.0001)$ similar to walking on a treadmill with moderate intensity $(-16.9 \pm 3.3 \mathrm{mmHg}, p<0.0001)$, whereas the control group without exercise did not demonstrate a blood pressure reduction. The sedentary video game also demonstrated a reduction in systolic blood pressure $(-10.0 \pm 3.1 \mathrm{mmHg}, p=0.0039)$, statistically similar to the active video game and walking on a treadmill with similar intensity to the active video game $(8.7 \pm 2.9 \mathrm{mmHg}, p=0.0034)$. Regarding the diastolic component, the active video game $(-7.6 \pm 1.5 \mathrm{mmHg}, p<0.0001)$, walking on a treadmill with moderate intensity $(-8.3 \pm 2.9 \mathrm{mmHg}, p=0.0042)$, and the sedentary video game $(-7.5 \pm 2.7 \mathrm{mmHg}, p=0.0098)$ produced similar reductions in diastolic blood pressure. The active video game was a procedure that promoted a sympathetic-vagal balance reduction from pre-exercise to 60 minutes post exercise $(4.2 \pm 0.8$ to $2.3 \pm 0.5, p=0.0486)$.
\end{abstract}

Conclusions. Active video game play promoted a similar reduction in blood pressure compared to traditional walking exercise with the advantage of promoting reduction in the sympathetic inflow to the heart.

Key words: post exercise hypotension, blood pressure, video games, exercise, chronic disease, health

\section{Introduction}

A recent trend in the market has been the development of active video games (AVG). Active video games are similar to traditional video games, with the difference being that the player needs to move in order to move the characters on the screen, instead of simply manipulating control buttons or a joystick [1]. By incorporating physical activity, these games could attenuate the negative effects of sedentary behavior promoted by traditional video games [2].

Previous studies show that AVGs generate low physiological demand, between $20 \%$ and $44 \%$ of the maxi- mum heart rate (MHR) [3-6]. Only some types of games at greater difficulty levels can produce increased levels of physical activity and significant changes in physiological variables such as heart rate (HR), energy expenditure, and oxygen uptake. Four studies showed intensity between $50 \%$ and $70 \%$ of MHR in young people who played AVG [3, 7-9].

Assuming that consensus statements and medical society guidelines and exercise [10] indicate that the optimal intensity for promoting metabolic exercise adaptations and health benefits is $60-85 \%$ of MHR, AVGs would only be beneficial to the health of people with low fitness levels. In fact, many studies have been

Correspondence address: Alexandre Sergio Silva, Federal University of Paraíba, Paraíba, Brazil,

e-mail: alexandresergiosilva@yahoo.com.br

Received: November 19, 2016

Accepted for publication: January 10, 2018

Citation: da Silva TF, de Franca ACL, de Souza MF, Silva AS. A single session of active video game play promotes post-exercise hypotension in hypertensive middle-aged subjects. Hum Mov. 2018;19(2):82-89; doi: https://doi.org/10.5114/hm.2018.74063. 
conducted with individuals with critically low physical fitness levels in rehabilitation after heart surgery [11] and physiotherapy [12], or overweight [13] and obese populations [14].

For anti-hypertensive treatment purposes, aerobic exercise has been found to be an effective non-pharmacological tool. While it is recognized by the European Society of Hypertension and the European Society of Cardiology [15] that moderate-intensity exercise is the most effective for promoting blood pressure reduction, there is evidence that low-intensity exercise such as $30 \%$ to $60 \%$ of maximum oxygen uptake or heart rate reserve are also capable of promoting acute reduction of blood pressure (BP) after exercise performance [16, 17]; a phenomenon called post-exercise hypotension (PEH) [17, 18].

Faced with these facts, this research was designed to test the hypothesis that an AVG session is capable of decreasing $\mathrm{BP}$, so that this type of entertainment can be indicated as an alternative to anti-hypertensive treatment. Therefore, the aim of this study was to determine if an AVG session is capable of promoting $\mathrm{PEH}$ in comparison to a classic aerobic exercise session.

\section{Material and methods}

\section{Study volunteers}

Fifteen (15) hypertensive patients of both genders ( $49.0 \pm 1.6$ years old) participated in this study, 5 men and 10 women. Most used more than one antihypertensive drug (drug multitherapy) and had controlled blood pressure during the study period (below 140/90 $\mathrm{mmHg}$ ). They maintained the medication throughout the study period. They had a mean BMI in the range between overweight and obesity grade I, and blood glucose correlated to insulin resistance, although none had diagnosed diabetes (Table 1). These characteristics were the result of prior application of the following inclusion criteria: 1) being hypertensive; 2) being classified as middle-aged (40 to 59 years); 3) being overweight or obese of degree I; 4) not performing regular exercise; 4) not using beta-blocker drugs or calcium channel blockers; 5) not being affected by labyrinthitis; 6) women not in menopause; and 4) not already being practitioners of any AVGs. The following were considered as exclusion criteria: 1) modifying antihypertensive treatment during treatment; 2) not developing the ability to play during the adaptation period; 3) not performing all procedures of the study.

The volunteers were at the university hospital and health center located in the vicinity of the laboratory where the study was conducted. Twenty people attended the invitation and were included in the inclusion criteria, five of whom did not complete all the experimental procedures.

\section{Study design}

The subjects were submitted to five randomly ordered protocols (www.randomizer.com) lasting $60 \mathrm{~min}$ utes: 1 - AVG (Dance Dance Revolution ${ }^{\circledR}$ ); 2 - Walking/running while reproducing the intensity obtained in the AVG session (WGI); 3 - Walking/running with an intensity between $60 \%$ and $85 \%$ of MHR (WMI); 4 - sedentary video game (SVG); and 5 - A session control without exercise $(\mathrm{CON})$, where participants remained at rest. The session order was randomly determined and was were carried out with a minimum of 48 hours between each session. Intensity was monitored by HR and PE measurements. BP and cardiac autonomic modulation (CAM) were assessed before and for a period of 60 minutes after exercise, at intervals of 10 minutes between each measurement (Figure 1).

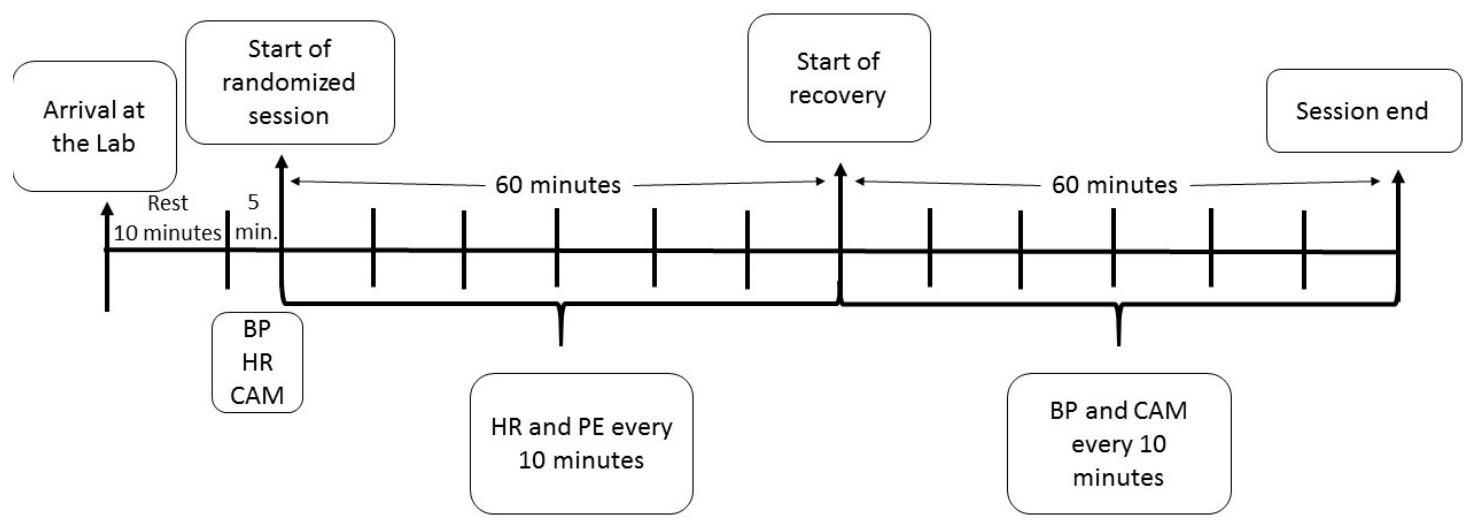

Figure 1. Experimental session design 
T.F. da Silva, A.C.L. de Franca, M.F. de Souza, A.S. Silva, Active video game play and hypertension

Adaptation to the game and instructions before the study

Volunteers underwent an adaptation period which included three 20-minute sessions before starting the experimental protocols. In these sessions, they were instructed in the proper way of handling the AVG and SVG instruments and were informed about the different arrow directions used in the games. These three sessions proved sufficient for the participants to understand the dynamics of the two games and allowed them to gain enough coordination to play without interruption.

After the adaptation period, a schedule was made with the volunteers and they were instructed not to eat/drink anything caffeinated/alcoholic for at least 48 hours before each experimental session.

\section{AVG and SVG procedures}

The AVG used in the study was Dance Dance Revolution ${ }^{\circledR}$ (SSD Company Ltd., Shiga, Japan) for Playstation ${ }^{\circledR}$ (San Mateo, CA, USA). In this AVG, the player/subject simulates synchronized dance steps on a pressure-sensitive pad corresponding to the order on the screen, replacing the traditional buttons of a SVG control/joystick. The music style in the game is similar to those used in gyms. The game consists of arrows passing in four directions which appear on the screen randomly and in the rhythm of different songs contained in the game. When the arrows show up on the screen, the player must step on the corresponding arrow on the pad in conjunction with the music rhythm. At the end of each song, the next song was immediately selected to minimize the time interval between them. A multimedia projector used as the screen was connected to the video game, where the game image being played was shown.

The SVG game was the same as the AVG, with the only difference being that the mat was replaced by a joystick, and volunteers remained seated during the game. Both AVG and SVG sessions lasted for 60 minutes. Heart rate was measured every 10 min using a heart rate monitor (RS800CX Polar ${ }^{\circledR}$, Polar ElectroOy, Kempele, Finland).

\section{Aerobic exercise protocol}

The subjects performed two aerobic exercise sessions lasting 60 minutes on a treadmill (Moviment ${ }^{\circledR}$ LX160i - Brudden LTDA, Amazon, Brazil). One session was performed at an intensity similar to the intensity reached during the AVG session, and the other session was performed with moderate intensity (ranging between $60 \%$ to $85 \%$ of maximum heart rate reserve).

Heart rate was monitored by a heart rate monitor (RS800CX Polar ${ }^{\circledR}$, Polar ElectroOy, Kempele, Finland) every 10 minutes to ensure that volunteers maintained the predetermined intensity during exercise on the treadmill. For the exercise protocol on the treadmill with AVG intensity, the lowest and highest measured heart rate found in the video game was adopted as a target zone for the heart rate on the treadmill. For the moderate intensity protocol, the target heart rate was determined according to an equation (Equation 1) proposed by Karvonen et al. [19]:

Equation I

$F C T=F C R+i \%(F C M-F C R)$,

$F C T=$ heart rate training; $F C R=$ heart rate at rest; $F C M=$ Maximum heart rate; $i \%=$ training intensity

During the rest period, the Borg Subjective Perception of Effort Scale [20] was presented to the subjects with indices 6-20 for the subjects to become acquainted with fatigue stages that ranged from very mild to exhaustive. Perceived effort (PE) was questioned every 10 minutes during the games or exercise on the treadmill.

\section{Blood pressure monitoring}

After the subjects arrived at the data collection site, they were instructed to remain seated for 10 minutes in an illuminated quiet room with controlled temperature between $295.1^{\circ} \mathrm{C}$ and $298.1^{\circ} \mathrm{C}$, and baseline BP was then measured. New BP measurements were taken immediately after the end of exercise and 60 minutes post exercise, and procedures were taken every $10 \mathrm{~min}$ utes post-recovery. BP was measured by auscultation, strictly following the protocol proposed in the VI Brazilian Guidelines on Hypertension [21]. An aneroid sphygmomanometer (WelkinAlllyn ${ }^{\circledR}$, Skaneateles Falls, New York, USA) was used, with an accuracy of two millimeters of mercury and previously calibrated against a mercury column and a Premium ${ }^{\circledR}$ stethoscope (G-Tech).

\section{Cardiac Autonomic Modulation (CAM)}

CAM was determined by recording the variability RR interval of HR, using the RS800CX Polar ${ }^{\circledR}$ heart rate monitor (Polar ElectroOy, Kempele, Finland). CAM was recorded after 10 minutes of initial rest and 
recovery post treadmill or video game procedure, every 10 minutes for 60 minutes. Each measure lasted five minutes. Data was transported through an infrared device to a computer equipped with software by the same manufacturer. The data was analyzed in the frequency domain (sympathetic-vagal balance - LF/HF) in Kubios software (University of Eastern Finland, Kuopio, Finland).

\section{Statistical treatment}

Data was initially tested for normality and homogeneity by the Shapiro-Wilks and Levene tests, respectively. A one way ANOVA test was then applied to compare the baseline for HR and BP within five days of procedures, and to compare intra-session data. A repeated measures ANOVA test was then applied to evaluate possible differences in BP responses and CAM between procedures. The adopted confidence level was $95 \%$ or $p<0.05$, and 3.0 Instat software was also used for Statistical analysis (GraphPad Instat, San Diego, CA, USA).

\section{Ethical approval and informed consent}

This study was approved by the Ethics in Research Committee of the Lauro Wanderley University Hospital under protocol $n^{\circ}$ 201/11. The subjects were then asked to sign the Free and Informed Consent form (TFIC) after they had been cleared for all study procedures, according to the Resolution 196/96 of the National Health Council. In addition, this study followed all guidelines in the Helsinki Declaration (1964).

\section{Results}

In the period prior to the five experimental procedures, BP, HR and sympathetic-vagal balance values were similar (Table 1). In this table, it can be observed that the hypertensive individuals demonstrated controlled blood pressure in the moments prior to the beginning of all the experimental procedures.

HR remained around $80 \mathrm{bpm}$ in the control procedure and varied only $80.7 \pm 2.2$ beats $\cdot \mathrm{min}^{-1}$ to 86.9 \pm 3.4 beats $\cdot \mathrm{min}^{-1}$ in SVG. The AVG procedure caused an increase in HR from $82.2 \pm 2.8$ beats $\cdot \mathrm{min}^{-1}$ to 95.0 \pm 4.4 beats $\cdot \mathrm{min}^{-1}$, which was equivalent to $13.1 \%$ of the MHR, as can be seen in Figure 2(A). This was successful in simulating the walking exercise intensity of the game, as HR increased to values similar to those measured in the AVG procedure without statistical differences in any of those moments. Meanwhile, volunteers reached $129.2 \pm 4.6$ beats $\cdot \mathrm{min}^{-1}$ in the WMI, which corresponded to $52.3 \%$ of MHR.

In Figure 2(B), it can be observed that the volunteers reported between 6 and 10 on the PE scale for the AVG session, while they reported between 9 and 14 in the moderate exercise session. For HR, the WGI session was similar to an AVG session, and volunteers reported

Table 1. Hemodynamic and clinical characteristics of the participants

\begin{tabular}{|c|c|c|c|c|c|}
\hline \multicolumn{3}{|l|}{ Variables } & \multicolumn{3}{|c|}{$\mathrm{x} \pm \mathrm{SD}$} \\
\hline \multicolumn{3}{|l|}{ Age (years) } & \multicolumn{3}{|c|}{$49.0 \pm 1.6$} \\
\hline \multicolumn{3}{|l|}{ Gender (man/woman) } & \multicolumn{3}{|c|}{$5 / 10$} \\
\hline \multicolumn{3}{|l|}{$\mathrm{BMI}\left(\mathrm{kg} \cdot \mathrm{cm}^{2}\right)$} & \multicolumn{3}{|c|}{$30.8 \pm 1.1$} \\
\hline \multicolumn{3}{|l|}{$\mathrm{WC}(\mathrm{cm})$} & \multicolumn{3}{|c|}{$98.5 \pm 1.9$} \\
\hline \multicolumn{3}{|l|}{ Glycemia $\left(\mathrm{mg} \cdot \mathrm{dl}^{-1}\right)$} & \multicolumn{3}{|c|}{$115.2 \pm 8.0$} \\
\hline \multicolumn{3}{|c|}{ Total Cholesterol (mg · dl $\left.\mathrm{l}^{-1}\right)$} & \multicolumn{3}{|c|}{$213.7 \pm 16.0$} \\
\hline \multicolumn{3}{|c|}{ HDL Cholesterol (mg $\left.\cdot \mathrm{dl}^{-1}\right)$} & \multicolumn{3}{|c|}{$47.2 \pm 5.2$} \\
\hline \multicolumn{3}{|c|}{ LDL Cholesterol (mg · dl $\left.{ }^{-1}\right)$} & \multicolumn{3}{|c|}{$169.9 \pm 14.7$} \\
\hline \multicolumn{3}{|c|}{ Triglycerides $\left(\mathrm{mg} \cdot \mathrm{dl}^{-1}\right)$} & \multicolumn{3}{|c|}{$255.2 \pm 61.7$} \\
\hline \multicolumn{3}{|c|}{ Drug monotherapy/multitherapy } & \multicolumn{3}{|c|}{$40 \% / 60 \%$} \\
\hline & AVG & WMI & SVG & WGI & $\mathrm{CON}$ \\
\hline HRR (beats $\cdot \min ^{-1}$ ) & $82.2 \pm 2.8$ & $81.1 \pm 2.7$ & $80.7 \pm 2.2$ & $75.3 \pm 2.5$ & $81.8 \pm 2.8$ \\
\hline SBPR (mmHg) & $126.9 \pm 2.7$ & $127.7 \pm 3.1$ & $125.9 \pm 2.5$ & $119.7 \pm 3.7$ & $120.5 \pm 4.6$ \\
\hline DBPR (mmHg) & $81.7 \pm 2.5$ & $83.6 \pm 1.7$ & $84.6 \pm 1.1$ & $79.5 \pm 2.6$ & $78.6 \pm 2.3$ \\
\hline $\mathrm{LF} \cdot \mathrm{HF}$ & $4.2 \pm 0.8$ & $2.9 \pm 0.6$ & $3.9 \pm 1.0$ & $3.2 \pm 0.9$ & $2.4 \pm 1.2$ \\
\hline
\end{tabular}

Data is mean and standard error of the mean. $\mathrm{x} / \mathrm{SD}$ : means and standard deviation of mean BMI = body mass index; $\mathrm{WC}=$ waist circumference; $\mathrm{HDL}=$ high density lipoprotein; $\mathrm{LDL}=$ low density lipoprotein; $\mathrm{HRR}=$ heart rate at rest; $\mathrm{SBPR}=$ systolic blood pressure at rest; $\mathrm{DBPR}=$ diastolic blood pressure at rest;

$\mathrm{LF} \cdot \mathrm{HF}=$ sympathetic-vagal balance. No statistical difference for $p<0.05$ (one way ANOVA). 
T.F. da Silva, A.C.L. de Franca, M.F. de Souza, A.S. Silva, Active video game play and hypertension

A

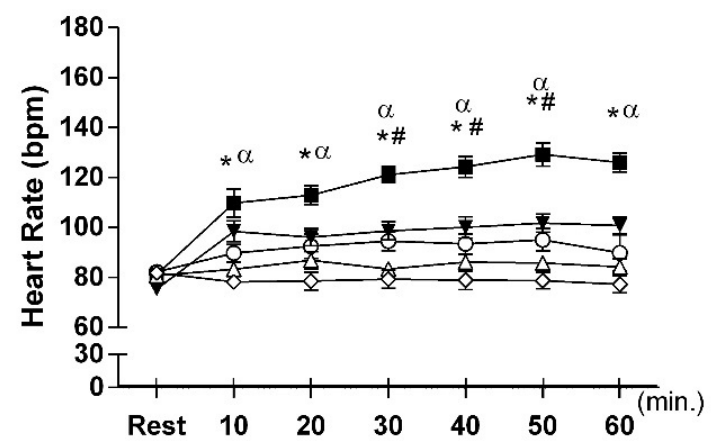

B

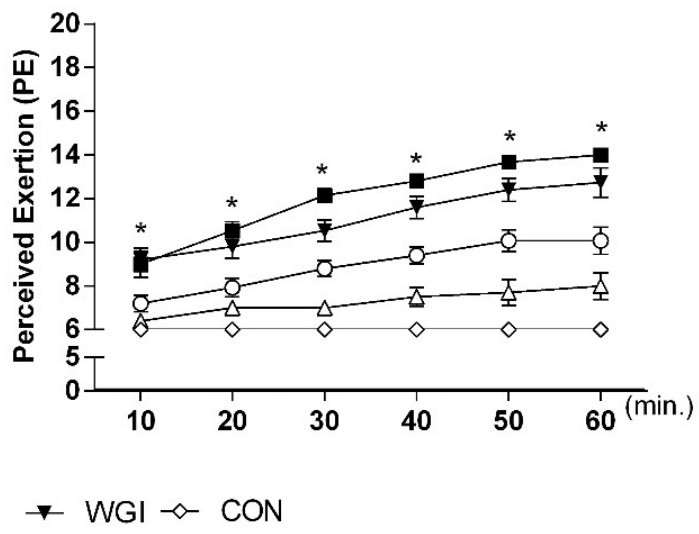

Figure 2. Heart rate values (FC) (A) and perceived exertion (PE) (B) in all five procedures measured every 10 minutes. Data is mean and standard error of the mean. For HR (panel A), ${ }^{*}=$ significant difference between the WMI session and other sessions. \# = Significant difference between AVG session and the session control. $\alpha=$ Significant difference between WMI session in the moments 10 to 60 minutes and rest moment. For PE (panel B), ${ }^{*}=$ significant difference between WMI and WGI sessions and other results of the study. $(p<0.05)$; Repeated measures ANOVA
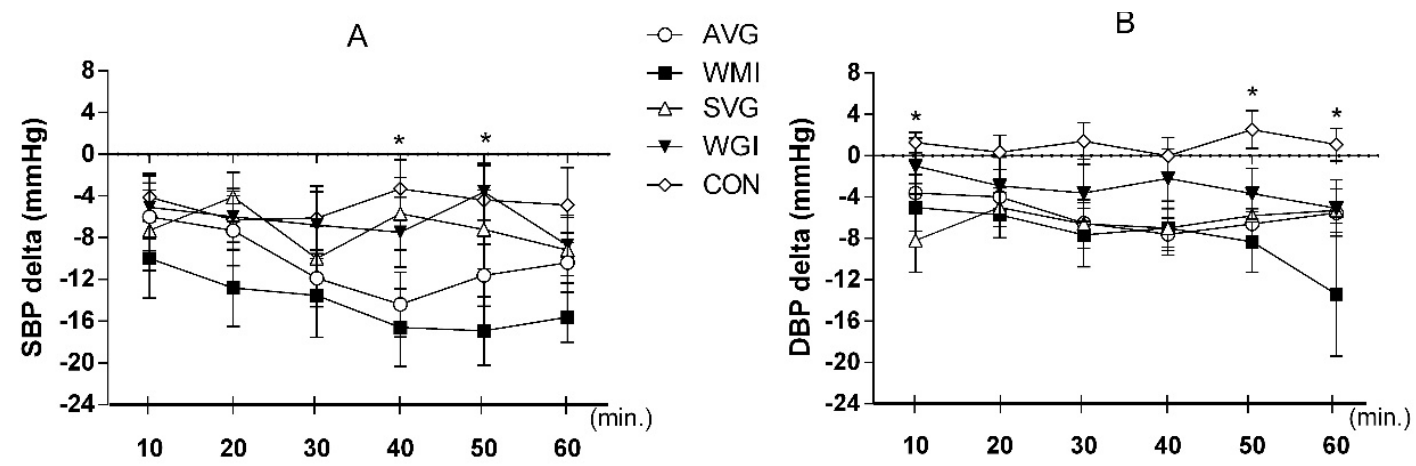

Figure 3. Blood pressure delta values for systolic (A) and diastolic (B) components during the recovery period for each of the experimental procedures performed. Data is mean and standard error of the mean. ${ }^{*}=$ Significant difference between the WMI session and the CON session after 40 minutes and WMI and WGI, 50 minutes (systolic BP) and between WMI and CON at 50 and 60 min (diastolic BP) and significant difference between the session WMI and absence of PEH (zero) for both components of the BP. $(p<0.05)$; Repeated measures ANOVA

greater feelings of fatigue in the walking procedure compared to the AVG session.

Exercise protocol at moderate intensity resulted in significant systolic PEH at all times when BP was measured in the post-exercise recovery period. Interestingly, this was the same behavior after the AVG session. Although the descriptive values of systolic PEH after the WMI session were shown to be larger relative to the AVG session (as can be seen in Figure 3), statistically significant differences were not observed between WMI and AVG sessions along the post exercise recovery period. Furthermore, WMI resulted in systolic PEH up to $16.9 \pm 3.3 \mathrm{mmHg}$ at 50 minutes post exercise, and post AVG systolic PEH reached $14.4 \pm 3.0 \mathrm{mmHg}$ at 40 minutes. There was a sig- nificant PEH for SVG and WGI, but only at 30 and 60 minutes (to SVG), and at 30, 40 and 60 minutes of the recovery period (for WGI).

A similar response was observed for the diastolic component, where WMI and AVG resulted in significant PEH at all times after exercise. The magnitude of PEH was very similar between these two procedures until 50 minutes, so that no statistical differences were observed $(\mathrm{AVG}=7.6 \pm 1.5 \mathrm{mmHg}$, $\mathrm{WMI}=$ $7.5 \pm 2.7 \mathrm{mmHg}$ ). Regarding measurements taken 60 minutes post exercise, PEH post WMI increased sharply to $13.4+6.0 \mathrm{mmHg}$, while it remained unchanged post AVG $(-5.5 \pm 3.3 \mathrm{mmHg})$. The SVG session resulted in a similar behavior to AVG and WMI sessions, which was a significant PEH at all times of 


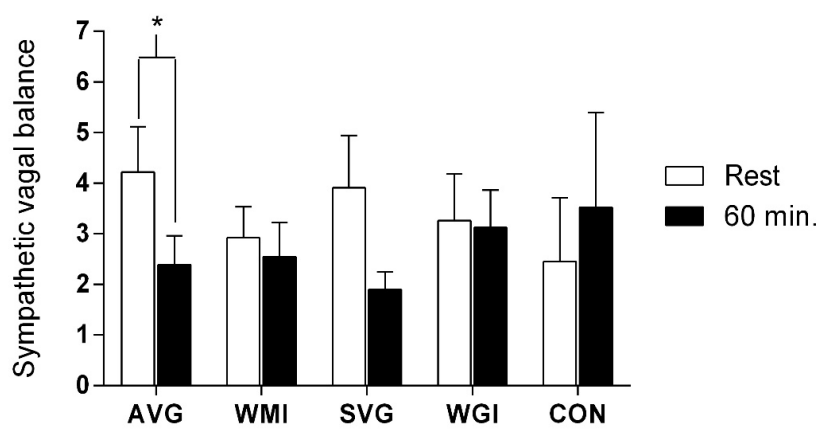

Figure 4. Behavior of sympathetic-vagal balance (LF/HF) in each of the procedures, at rest and after 60 minutes of the recovery period. Data is mean and standard error of the mean. ${ }^{*}=$ Significant difference between rest and 60 minutes of recovery in AVG session $(p=0.04)$; Repeated measures ANOVA

the BP measurement after exercise. Meanwhile, a significant $\mathrm{PEH}$ was only observed at 60 minutes post-exercise with a similar intensity to AVG (WGI) (Figure 3).

Sympathetic-vagal balance is shown in Figure 4. A significant reduction occurred for the AVG session $(4.2 \pm 0.8$ and $2.3 \pm 0.5)$ from pre to 60 minutes post procedure. This phenomenon did not occur for any of the other procedures. However, a reduction of sympathetic-vagal balance tended to occur in the SVG procedure (3.9 \pm 1.0 and $1.8 \pm 0.3$ from pre to $60 \mathrm{~min}$ utes post procedure, $\mathrm{p}=0.053$ ).

\section{Discussion}

Data from this study shows that an AVG session promotes a pressure reduction in both systolic and diastolic components, similar to a moderate intensity aerobic exercise session. This BP reduction was accompanied by a reduction of the sympathetic-vagal balance. The SVG session also promoted some PEH, but in a very discreet manner in relation to AVG.

PEH occurred in the AVG procedure, despite this activity having been performed with an intensity of only $13.1 \%$ MHR. Interestingly, the magnitude of PEH was similar to that encountered in the moderate intensity exercise procedure of this study and to what has been reported in the literature for aerobic exercise protocols with an intensity between $50 \%$ and $80 \%$ of MHR (reductions of 18 to $20 \mathrm{mmHg}$ for systolic and 7-9 mmHg for diastolic BP) [22]. In addition, $\mathrm{PEH}$ values for AVG were higher than what has been reported for a resistance exercise modality $(2-4 \mathrm{mmHg}$ for systolic when to diastolic BP) [23, 24].

The main finding of this study is that a low demand of physical effort is required to promote $\mathrm{PEH}$ similar to a traditional session of aerobic exercise. Although this is the first time that AVG practice was able to promote a hypotensive response, there are already reports of studies in which very light intensity activities such as Hatha Yoga resulted in both PEH $(10 \mathrm{mmHg}$ and $5 \mathrm{mmHg}$ for systolic and diastolic components) and a reduction of cardiac sympathetic activity in hypertensive subjects [25]. Another study showed reduced cardiac sympathetic nervous system activity after taichi-chuan practice [26].

Although moderate intensity is considered ideal to promote PEH [21], there are previous studies in which low-intensity aerobic exercise (cycle ergometer and treadmill) $\left(30 \%\right.$ to $50 \%$ of peak $\left.\mathrm{VO}_{2}\right)$ has also promoted PEH [27]. In view of this perspective, exercise performed in the intensity of the game (WGI) should also similarly promote PEH. However, this was not the phenomenon observed in our data. Thus, another factor must be contributing to explain the phenomenon observed in the present study. One plausible hypothesis is that AVG may have promoted feelings of pleasure, which may have caused increased psychological relaxation and consequent deactivation of vasoconstriction factors. The basis for this possibility is the fact that the AVG was the only procedure that promoted a reduction in cardiac heart rate sympathetic activity, which can reduce sympathetic nerve activity in the heart and vessels, as stated by Van Lieshout et al. [28].

A previous study showed that the practice of AVG led to a reduction in systolic $\mathrm{BP}$ at $4 \mathrm{mmHg}$, and no change in diastolic blood pressure 40 minutes after AVG in children [5]. However, until this study (at least to our knowledge), studies including adults and hypertensive subjects were not encountered in the literature. Thus, data from this study only represents the beginning of a line of research which should be expanded. Therefore, some questions remain to be explored. First, it seems necessary to verify if the durability of $\mathrm{PEH}$ will be the same as that found after traditional exercise. The literature reports that aerobic exercise promotes PEH in short periods, such as 60 and 120 minutes [29], and may reach up to 22 hours [16]. In order to answer if AVG also results in the same time duration of $\mathrm{PEH}$, it is necessary for future studies to be carried out with 24-hour ambulatory measurements in addition to clinical BP measurements.

The intensity found for the exercise and AVG sessions in the present study should be weighed by the fact that it was determined from an estimation equation. In fact, Robert and Robegs warn that estimation equations of maximum heart rate present a considerable margin of error. In order to suppress this limitation in future studies, the ideal experiment is to per- 
T.F. da Silva, A.C.L. de Franca, M.F. de Souza, A.S. Silva, Active video game play and hypertension

form a maximum effort test for direct determination of maximum heart rate [30]. Another limitation is that the intensity of the AVG is a key question to understand the therapeutic capacity of this game, but we did not evaluate the metabolic demand imposed by this game.

Taken together, this study has shown that an AVG session promotes PEH similar to that achieved by traditional walking exercise of moderate intensity. The practical implications of this study are that AVG practice can be configured as a new alternative to physical activity as a non-pharmacological tool for treatment of hypertension. Aspects of pleasure, the ability to hold attention, and the possibility to be practiced at home, alone or with friends make this form of physical activity interesting and may increase the chances of people with hypertension adopting domestic attitudes to increase physical activity as an antihypertensive treatment. However, our data does not allow for exploration of the mechanisms that may explain the $\mathrm{PEH}$ promoted by AVG to the extent and depth necessary, thus further research needs to be conducted to explore both pleasure scales and physiological parameters of pleasure and well-being, including a comparison between the production of endorphins in practicing active video games and traditional exercises.

\section{Acknowledgements}

Not applicable.

\section{Disclosure statement}

No author has any financial interest or received any financial benefit from this research.

\section{Conflict of interest}

The authors state no conflict of interest.

\section{References}

1. Staiano AE, Calvert SL. The promise of exergames as tools to measure physical health. Entertain Comput. 2011 Jan 1;2(1):17-21, doi: 10.1016/j.entcom.2011.03.008.

2. Weaver JB 3rd, Mays D, Sargent Weaver S, Kannenberg W, Hopkins GL, Eroglu D, et al. Health-risk correlates of video-game playing among adults. Am J Prev Med. 2009 Oct;37(4):379-380; doi: 10.1016/j.amepre. 2009.06.014.

3. Siegel SR, Haddock BL, Dubois AM, Wilkin LD. Active video/arcade games (exergaming) and energy expenditure in college students. Int J Exerc Sci. 2009; 2(3):165-174.

4. Douris PC, McDonald B, Vespi F, Kelley NC, Herman L. Comparison betweem Nintendo Wii Fit aerobics and tradicional aerobic exercise in sedentary young adults. JSCR. 2012;26(4):1052-1057; doi: 10.1519/JSC.0b013e $31822 \mathrm{e} 5967$.
5. Rauber SB, Carvalho FC, Souza IRC, Mazzoccante RP, Franco CBS, Farias DL, et al. Cardiovascular variables during and after the practice of video game active „Dance Dance Revolution“ and television [Variáveis cardiovasculares durante e após a prática de víde game ativo “Dance Dance Revolution” e televisão, in Portuguese]. Motriz. 2013;19(2):358-367, doi: 10.1590/S1980-657 42013000200013.

6. Mills A, Rosemberg M, Stratton G, Carter HH, Spence AL, Pugh CJ, et al. The effect of exergaming on vascular function in children. J Pediat. 2013;163(3):806-810; doi: 10.1016/j.jpeds.2013.03.076.

7. Graves LEF, Ridgers ND, Stratton G. The contribution of upper limb and total body movement to adolescents' energy expenditure whilst playing Nintendo Wii. Eur J Appl Physiol. 2008;104(4):617-623; doi: 10.1007/ s00421-008-0813-8.

8. O'Donovan C, Hussey J. Active video game as a form of exercise and the effect of gaming experience: a preliminary study in healthy young adults. Physiotherapy. 2012; 98(3):205-210. doi: 10.1016/j.physio.2012.05.001.

9. Mellecker RR, McManus AM. Active video games and physical activity recommendations: a comparison of the Gamercise Stepper, XBOX Kinect and XaviX J-Mat. J Sci Med Sport. 2014;17(3):288-292, doi: 10.1016/j. jsams.2013.05.008.

10. Garber CE, Blissmer B, Deschenes MR, Franklin BA, Lamonte MJ, Lee IM, et al. Quantity and quality of exercise for developing and maintaining cardiorespiratory, musculoskeletal, and neuromotor fitness in apparently healthy adults: guidance for prescribing exercise. Med Sci Sports Exerc. 2011;43(7):1334-1359; doi: 10.1249/MSS.0b013e318213fefb.

11. Broeren J, Claesson L, Goude D, Rydmark M, Sunnerhagen KS. Virtual rehabilition in an activity center for community-dwelling persons with stroke. The possibilities of 3-dimensional computer games. Cerebrovasc Dis. 2008;26(3):289-296; doi: 10.1159/000149576.

12. Burk JW, McNeill MDJ, Charles DK, Morrow PJ, Crosbie JH, McDonough SM. Serious Games for Upper Limb Rehabilitation Following Stroke. In: 1st IEEE International Conference in Games and Virtual Worlds for Serious Applications VS-GAMES ‘09; 2009 Mar 23-24; Coventry, UK. 2009. p. 103-110; doi: 10.1109/VSGAMES.2009.17.

13. Adamo KB, Rutherford JA, Goldfield GS. Effects of interactive video game cycling on overweight and obese adolescent health. Appl Physiol Nutr Metab. 2010 Dec; 35(6):805-815; doi: 10.1139/H10-078.

14. Goldfield GS, Kenny GP, Hadjiyannakis S, Phillips P, Alberga AS, Saunders TJ, et al. Video game playing is independently associated with blood pressure and lipidis in overweight and obese adolescents. Plos One. 2011;6(11): e26643; doi: 10.1371/journal.pone.0026643.

15. Task Force Members, Mancia G, Fagard R, Narkiewicz K, Redón J, Zanchetti A, et al. 2013 ESH/ESC Guidelines for the management of arterial hyperten- 
sion: the Task Force for the management of arterial hypertension of the European Society of Hypertension (ESH) and of the European Society of Cardiology (ESC). Eur Heart J. 2013;34(28):2159-2219; doi: 10.1093/ eurheartj/eht151.

16. Laterza MC, Brandão Rondon MUP, Negrão CE. Antihypertensive effect of exercise [Efeito anti-hipertensivo do exercício, in Portuguese]. Rev Bras Hipert. 2007; 14(2):104-111.

17. Medina FL, Lobo FS, Souza DR, Kanegusuku H, Forjaz CLM. Physical activity: impact on blood pressure [Atividade física: impacto sobre a pressão arterial, in Portuguese]. Rev Bras Hipertens. 2010;17(2):103-106.

18. Monteiro MF, Sobral Filho DC. Physical exercise and blood pressure control [Exercício físico e o controle da pressão arterial, in Portuguese]. Rev Bras Med Esporte. 2004;10(6):513-516; doi: 10.1590/S1517-869220040 00600008.

19. Karvonen MJ, Kental E, Mustala O. The effects of on heart rate a longitudinal study. Ann Med Exp Biol Fenn. 1957;35(3):307-315.

20. Noble BJ, Borg GA, Jacobs I, Ceci R, Kaiser P. A category-ration perceived exertion scale: relationship to blood and muscle lactates and heart rate. Med Sci Sports Exerc. 1983;15(6):523-528.

21. Sociedade Brasileira de Cardiologia, Sociedade Brasileira de Hipertensão, Sociedade Brasileira de Nefrologia. VI Brazilian Guidelines on Hypertension [VI Diretrizes Brasileiras de Hipertensão, in Portuguese]. Arq Bras Cardiol. 2010;95(1) Suppl 1:1-51.

22. Kelley GA, Kelley KS. Progressive resistence exercise and resting blood pressure: A meta-analisys of randomized controlled trials. Hypertension. 2000;35:838843; doi: 10.1161/01.HYP.35.3.838.

23. Mediano MFF., Paravidino V, Simão R, Pontes FL, Polito MD. Subacute behavior of blood pressure after power training in controlled hypertensives individuals [Comportamento subaguda da pressão arterial após treinamento de força em hipertensos controlados, in Portuguese]. Rev Bras Med Esporte. 2005;11(6):337-340; doi: 10.1590/S1517-86922005000600006.

24. Pinheiro CHJ, Medeiros RAR, Pinheiro DGM, Marinho MJF. Use of yoga as a non-pharmacological resource in the treatment of essential hypertension [Uso do ioga como recurso não farmacológico no tratamento da hipertensão essencial, in Portuguese]. Rev Bras Hipertens 2007;14(4):226-232.

25. Motivala SJ, Sollers J, Thayer J, Irwin MR. Tai Chi Chih acutely decreases sympathetic nervous system activity in older adults. J Gerontol A Biol Sci Med Sci. 2006; 61(11):1177-1180; doi:10.1093/gerona/61.11.1177.

26. Forjaz CL, Tinucci T, Ortega KC, Santaella DF, Mion D Jr, Negrão CE. Factors affecting post-exercise hypotension in normotensive and hypertensive humans. Blood Press Monit. 2000;5(5-6):255-262; doi: 10.1097/00126097200010000-00002.

27. Casonatto J, Polito MD. Hypotension after aerobic ex- ercise: a systematic review [Hipotensão pós exercício aeróbio: uma revisão sistemática, in Portuguese]. Rev Bras Med Esporte. 2012;15(2):151-157; doi: 10.1590/ S1517-86922009000200014.

28. Van Lieshout JJ, Wieling W, Karemaker JM, Eckberg DL. The vasovagal response. Clin Sci. 1991;81(5):575-586; doi.org/10.1042/cs0810575.

29. Forjaz CLM., Santaella DF, Rezende LO, Barreto ACP, Negrão CE. The duration of exercise determines the magnitude and duration of post-exercise hypotension [A duração do exercício determina a magnitude e a duração da hipotensão pós-exercício, in Portuguese]. Arq Bras Cardiol. 1998;70(2):99-104; doi: 10.1590/S0066782X1998000200006.

30. Robergs RA, Landwehr R. The surprising history of the "HRmax=220-age" equation. J Exerc Physiol online. 2002;5(2):1-10. 